\title{
Energy Usage and Carbon Emission Optimization Mechanism for Federated Data Centers
}

\author{
Dang Minh Quan ${ }^{1}$, Andrey Somov ${ }^{2}$, and Corentin Dupont ${ }^{2}$ \\ ${ }^{1}$ Institute of Information Technology for Economic, \\ National Economic University, Vietnam \\ quandm@upb. de \\ ${ }^{2}$ CREATE-NET, Trento, Italy \\ \{asomov, cdupont\}@create-net.org
}

\begin{abstract}
This work addresses the problem of high energy consumption and carbon emissions by data centers which support the traditional computing style. In order to overcome this problem we consider two allocation scenarios: single allocation and global optimization of available resources and propose the optimization algorithms. The main idea of these algorithms is to find a server in the data center with the lowest energy consumption and/or carbon emission based on current status of data center and service level agreement requirements, and move the workload there. The optimization algorithms are devised based on Power Usage Effectiveness (PUE) and Carbon Usage Effectiveness (CUE). The simulation results demonstrate that the proposed algorithms enable the saving in energy consumption from $10 \%$ to $31 \%$ and in carbon emission from $10 \%$ to $87 \%$.
\end{abstract}

Keywords: data center, traditional computing, power consumption, green computing, resource management.

\section{Introduction}

Until recently, the key performance indicator of a data center was its performance. However, the growing number of IT services and large scale tasks, resulting in higher power consumption [1] and carbon emission, have forced the ICT community to concern the energy efficiency of data centers carefully [2].

Several energy-aware approaches and resource management techniques have been introduced to tackle power consumption problem in the data center domain from different points of view. Many approaches are focused on workload consolidation in order to decrease the number of servers by switching them off/sleep and, therefore, to reduce the power consumption [3] [4]. Some techniques, in contrast, put efforts in finding the solutions of optimal workload placement in order to minimize the cooling systems energy consumption [5] while similar research works investigate the opportunity to reduce the power consumption of cooling systems by using intelligent scheduling or choosing the optimum temperature of cold air [7] [8]. The biological algorithm in [6] determines more power efficient servers within a data center facility and moves workload there.

J. Huusko et al. (Eds.): E2DC 2012, LNCS 7396, pp. 129-140, 2012.

(c) Springer-Verlag Berlin Heidelberg 2012 
The objective of this research work is twofold: to reduce the power consumption and carbon emission of a federated data center with the traditional mode of computation. To achieve this objective we propose the optimization algorithms for the single allocation of virtual machines and for global resources optimization.

The paper is organized as follows: Section 2 describes the problem formulation. Sections 3 and 4 present the algorithm for single allocation request and global optimization respectively. The simulation results based on different scenarios and the data centers configurations are shown in Section 5. Finally, we conclude and discuss our future work in Section 6.

\section{Problem Formulation}

We assume that we have a set of data centers $D$ including $N$ centers. Assume that in each data center $d_{l}$ we have a set of servers $S_{l}$. Each server $s_{l i} \in S_{l}$ is characterized with the number of cores and the amount of memory $\left(s_{l i} \cdot n r_{-}\right.$Core and $s_{l i} \cdot n r_{-} R A M$, respectively). As servers use central storage infrastructures (such as SAN, NAS or iSCSI external storage arrays), we do not have to care about storage in each server.

Each server $s_{l i}$ has a set of running virtual machines $V_{l i}$ including $k_{l i}$ virtual machines. Each virtual machine $v_{j} \in V_{l i}$ is characterized with required number of virtual CPU and amount of memory $\left(v_{j} \cdot r_{-} v C P U\right.$ and $v_{j} \cdot r_{-} R A M$, respectively) and the average CPU usage rate computed in $\%$ and the amount of memory $\left(v_{j} \cdot a_{-} U\right.$ Urate, $\left.v_{j} \cdot a_{-} R A M\right)$.

With each server $s_{l i}$ the following constraints (1)-(4) have to be met. The total usage rate of a certain number of CPUs on a certain number of VMs can not exceed the safe performance factor for this certain number of cores:

$$
\sum_{j=1}^{k_{l i}} v_{j} \cdot r_{-} v C P U * v_{j} \cdot a_{-} \text {Urate }<=k * s_{l i} \cdot n r_{-} \text {Core }
$$

where $k$ is the safe performance factor, $k<1$.

The average total memory used by the VMs on one server cannot exceed the amount of total available memory of the server:

$$
\sum_{j=1}^{k_{l i}} v_{j} \cdot a_{-} R A M<=s_{l i} \cdot n r_{-} R A M
$$

The total number of VMs with required number of virtual CPUs is less or equal to number of cores with predefined maximum number of virtual CPUs on board:

$$
\sum_{j=1}^{k_{l i}} v_{j} \cdot r_{-} v C P U<=s_{l i} \cdot n r_{-} \text {Core } * \max v C P U p \text { Core }
$$

where maxvCPUpCore is maximum number of virtual CPUs per Core.

The number of the server's VMs can not exceed the maximum number of VMs, maxVMpServer, allowed for the server:

$$
k_{l i}<=\max \text { VMpServer }
$$




\subsection{Allocation Request}

Assume we assign a new virtual machine with requirement ( $\left.v . r_{-} v C P U, v . r_{-} R A M\right)$ to server $s_{l i}$ as virtual machine $v_{k+l}$. The constraints above have to be met also with the new VM, so we have the following constraints.

$$
\begin{gathered}
\sum_{j=1}^{k_{l i}} v_{j} \cdot r_{-} v C P U * v_{j} \cdot a_{-} \text {Urate }+v_{k+1} \cdot r_{-} \nu C P U<= \\
<=k^{*} s_{l i} \cdot n r_{-} \text {Core } \\
\sum_{j=1}^{k_{l i}} v_{j} \cdot a_{-} R A M+v_{k+1} \cdot r_{-} R A M<=s_{l i} \cdot n r_{-} R A M \\
\sum_{j=1}^{k_{l i}} v_{j} \cdot r_{-} v C P U+v_{k+1} \cdot r_{-} v C P U<= \\
<=s_{l i} \cdot n r_{-}{ }^{C} \text { Core } * \max v C P U \text { CCOre } \\
k_{l i}+1<=\max \text { VMpServer }
\end{gathered}
$$

Before assigning the new VM to the server $s_{l i}$, its energy consumption, $E_{l i 0}$, is

$$
E_{l i 0}=f\left(U_{l i 0}\right)
$$

and CPU usage rate is

$$
U_{l i 0}=\frac{\sum_{j=1}^{k_{l i}} v_{j} \cdot r_{-} \nu C P U^{*} v_{j} \cdot a_{-} \text {Urate }}{s_{l i} \cdot n r_{-} \text {Core }}
$$

The total energy $T E_{l i 0}$, that data center used for the server $s_{l i}$ before assigning the new $\mathrm{VM}$ is

$$
T E_{l i 0}=P U E_{l} * E_{l i 0}
$$

The total $\mathrm{CO}_{2}$ emission, $C_{l i 0}$, due to the server $s_{l i}$ in the data center before assigning the new VM is

$$
C_{l i 0}=C U E_{l} * E_{l i 0}
$$

After assigning, the energy consumption is

$$
E_{l i 1}=f\left(U_{l i 1}\right)
$$

where CPU usage rate is 


$$
U_{l i 1}=\frac{\sum_{j=1}^{k_{l i}} v_{j} \cdot r_{-} v C P U^{*} v_{j} \cdot a_{-} \text {Urate }+v_{k+1} \cdot r_{-} v C P U}{s_{l i} \cdot n r_{-} \text {Core }}
$$

If the goal is in optimizing energy, we have to pick the server $s_{l i}$ in a way that minimizes $\left(E_{l i l}-E_{l i 0}\right)$.

$\mathrm{CO}_{2}$ emission is

$$
C_{l i 1}=C U E_{l} * E_{l i 1}
$$

If the goal is in optimizing $\mathrm{CO}_{2}$ emission, we have to pick the sever $s_{l i}$ in a way that minimizes $\left(C_{l i l}-C_{l i 0}\right)$.

\subsection{Global Optimization Request}

When moving a virtual machine $v_{l i}$ from center $d_{l}$ to center $d_{k}$, we have to consider the following constraints:

- Transfer time must be less than $T$

- Energy spent for the transfer must be less than $E_{t}$

After redistributing all running virtual machines over $D$, each server $s_{l i}$ has an updated set of running virtual machines $V_{l i l}$ including $k_{l i l}$ virtual machines. The new arrangement must also satisfy the above constraints (1)-(4).The total energy used in the federated data center is then

$$
E_{1}=\sum_{l=1}^{N} f\left(U_{l i 1}\right) \text { with } i \in[1, \mathrm{n}], l \in[1, \mathrm{~N}]
$$

where $n$ is the number of servers and $N$ is the number of data centers, $f\left(U_{l i l}\right)$, and CPU usage rate is as follows

$$
U_{l i 1}=\frac{\sum_{j=1}^{k_{i 1}} v_{j} \cdot r_{-} v C P U^{*} v_{j} \cdot a_{-} \text {Urate }}{s_{l i} \cdot n r_{-} \text {Core }}
$$

$\mathrm{CO}_{2}$ emission is

$$
C_{1}=\sum_{l=1}^{N} f\left(U_{l i 1}\right) * C U E_{l} \text { with } i \in[1, \mathrm{n}], j \in[1, \mathrm{~N}]
$$

We have to rearrange the workload in a way that minimizes the $\mathrm{CO}_{2}$ emission $C_{l}$.

If the goal is minimizing energy consumption, we also minimize $C_{l}$ but with $C U E_{l}$ replaced by $P U E_{l}$. 


\section{Algorithm for Single Allocation Request}

The main idea of the proposed algorithm is to go through each server in each data center to see the consumed energy and $\mathrm{CO}_{2}$ emission if we assign the VM to that server of the data center. From those collected data we will select the server of the data center having the smallest $\mathrm{CO}_{2}$ emission. The algorithm for minimizing energy is similar.

The algorithm for single allocation request (see Fig. 1) is presented in Table 1. In order to satisfy the VM allocation request the algorithm tries to find a server with minimum energy overhead. To do so, the algorithm takes into consideration the information about data center (data center description), including the CUE, and considers the acting SLA. Besides, the algorithm communicates with the power calculator for the evaluation of required power resources. Based on all these parameters, the algorithm, finally, proposes the candidate server for the allocation.

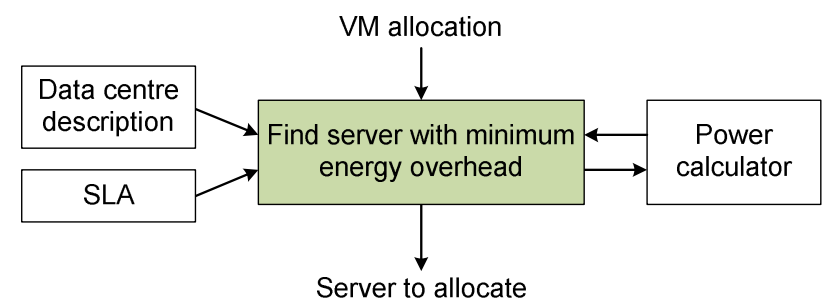

Fig. 1. Single allocation request diagram

The pseudo code provides the algorithm (see Table 1) explanation in more details and is as follows:

Table 1. Single allocation request for federated traditional data center

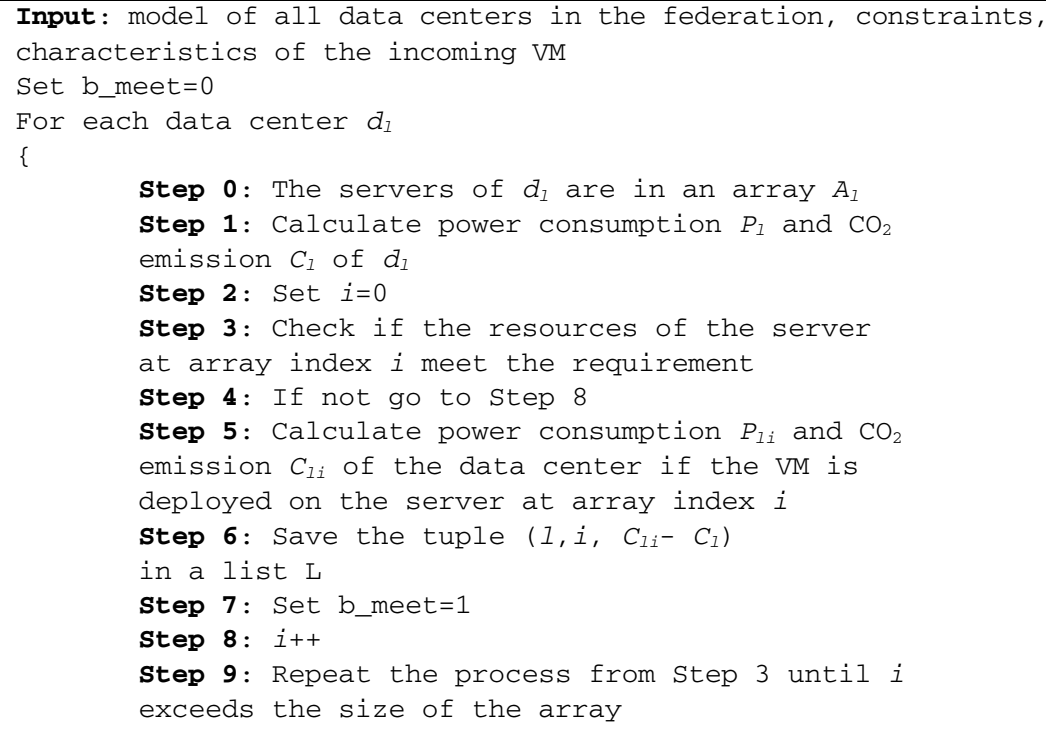


Table 1. (continued)

\begin{tabular}{|l|c|}
\hline 22 & If b_meet==1 \\
23 & Go through the list $L$ to find the tuple having \\
24 & the smallest $C_{1 i}-C_{1}$ \\
25 & Output: the server in the data center having \\
26 & the smallest $C_{1 i}-C_{1}$ \\
27 & If b_meet==0 \\
28 & Output: no solution \\
\hline
\end{tabular}

\section{$4 \quad$ Algorithm for Global Optimization Request}

This section describes the algorithm for global optimization request (see Fig. 2).

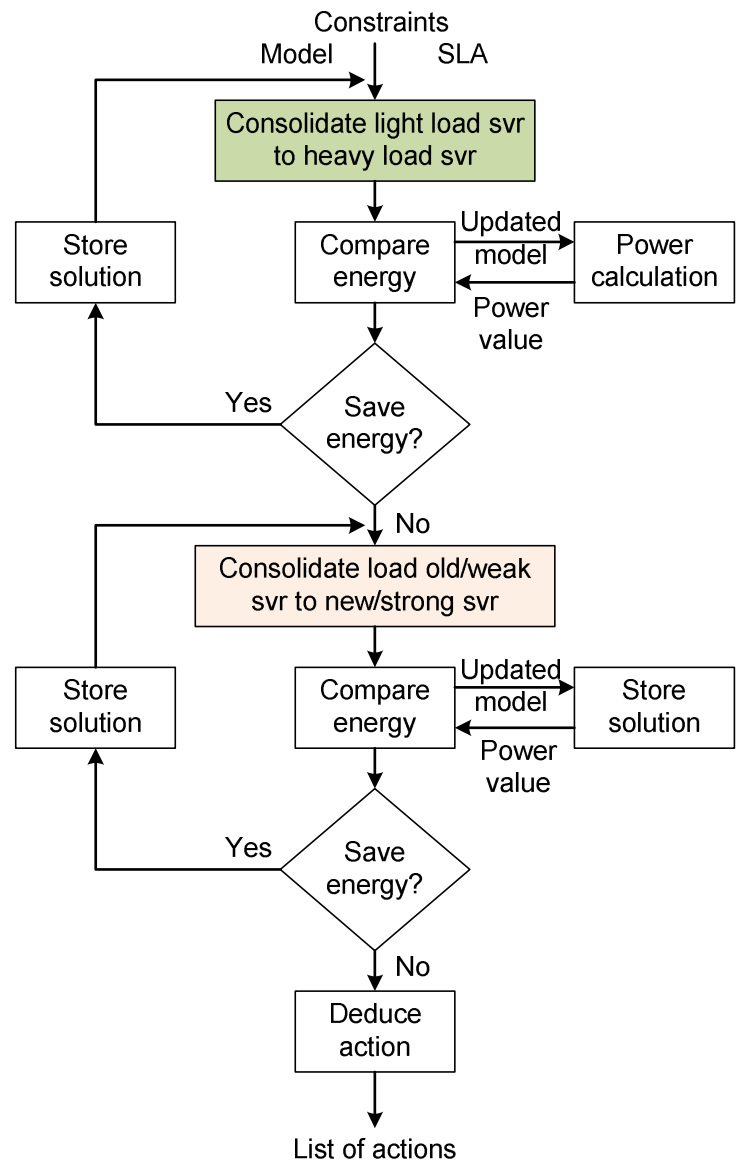

Fig. 2. Global optimization flow diagram 
The main idea of the algorithm is to move all the heavy loading VMs to the servers which have the best rate of $\mathrm{CO}_{2}$ emission or the largest computational horsepower. The VMs which cannot conveniently be moved out of the old data centers are rearranged inside the same data center to achieve the smallest possible $\mathrm{CO}_{2}$ emission value. The same mechanism can be applied to optimize the energy by replacing CUE by PUE.

The algorithm for global allocation request for federated traditional data center is as follows:

Table 2. Global allocation request for federated traditional data center

Input: model of all data centers in the federation, constraints step 0: Form list of all VMs $L V$ running in all data centers

step 1: Form list of all servers $L S$ in all data centers

step 2: Sort the list $L V$ in descending order of the actual CPU usage $A$.

The actual CPU usage $A$ of a VM $V$ is calculated with

$A=V \cdot r_{-} V C P U * V \cdot a_{-}$Urate

Step 3: Sort the list $L S$ in ascending order of the maximum $\mathrm{CO}_{2}$ emission $E$ per core of the server.

The maximum $\mathrm{CO}_{2}$ emission $E$ per core of the server $s$ is calculated with

$E=$ s.max_Power ${ }^{*} C U E / s . n r_{-}$Core

Step 4: For each server in the list $L S$, we use the traditional pack algorithm to reassign VMs in the list $L V$ to the server; then remove reassigned VMs out of $L V$

step 5: If there are still VMs in the list $L V$, we return the empty exchange list

Step 6: From the new assignment, form the exchange list

Output: Exchange list containing VMs that should be moved to other servers

Table 3. Pack algorithm for traditional data center

\begin{tabular}{|c|c|}
\hline 1 & Input: The server, list of VMs $L V$, constraints about transfer time \\
\hline 3 & Algorithm: \\
\hline 4 & Step 1: Determine the number of free resources in the server \\
\hline 6 & Step 2: (where nCPU and nMem is the number of $\mathrm{CPU}$ and size of RAM \\
\hline 7 & available on the server, respectively): \\
\hline 8 & For each VM in $L V$ \\
\hline 9 & \{ \\
\hline 10 & if $(($ fCPU*k $<$ nCPU*u_rate $)$ \\
\hline 11 & \{ \\
\hline 12 & Calculate the transfer time $T F$ from old data \\
\hline 13 & center to the one \\
\hline 14 & If $T F$ meets the transfer time constraints \\
\hline 15 & Put VM into list L1 \\
\hline 16 & Update the amount of free resources: \\
\hline 17 & fCPU $-=$ nCPU*u_rate \\
\hline 18 & fMem $-=$ nMem \\
\hline 19 & \} \\
\hline 20 & \} \\
\hline 21 & Output: L1 \\
\hline
\end{tabular}




\section{$5 \quad$ Simulation Results}

This section presents the simulation scenario and results for the federated traditional data center case.

\subsection{Simulation Scenario}

The simulation is done to study the saving rate of the resource allocation mechanism in different resource configuration scenarios. To do the simulation, we use 4 server classes with single core, duel cores, quad cores and six cores. The main parameters for each server class are presented in Table 4.

Table 4. Server configuration

\begin{tabular}{|c|l|l|l|c|l|l|l|l|}
\hline $\begin{array}{l}\text { Server } \\
\text { type } \\
(i)\end{array}$ & $\begin{array}{l}\text { Nr. } \\
\text { CPU }\end{array}$ & $\begin{array}{l}\text { Nr. } \\
\text { Cores }\end{array}$ & $\begin{array}{l}P_{\text {idle }} \text { CPU } \\
(\mathrm{W})\end{array}$ & $\begin{array}{l}f_{i} \\
(\mathrm{GHz})\end{array}$ & $\begin{array}{l}\text { RAM } \\
(\mathrm{MB})\end{array}$ & $\begin{array}{l}\text { Nr. } \\
\text { Fan }\end{array}$ & $\begin{array}{l}\text { Disk } \\
(\mathrm{MB})\end{array}$ & $\begin{array}{l}P_{\max } \\
(\mathrm{W})\end{array}$ \\
\hline 1 & 1 & 1 & 7.57 & 2.0 & 1000 & 4 & 400 & 102.22 \\
\hline 2 & 1 & 2 & 9.88 & 2.0 & 2000 & 4 & 500 & 103.39 \\
\hline 3 & 1 & 4 & 20.14 & 2.2 & 4000 & 5 & 800 & 171.70 \\
\hline 4 & 1 & 6 & 22 & 2.4 & 6000 & 6 & 1000 & 229 \\
\hline
\end{tabular}

We generated 3 resource configuration scenarios: modern data center, normal data center and old data center as in Table 5. In the normal data center the percentage of different server classes is fully balanced. In the old data center, the percentage of server classes with less cores is predominant.

Table 5. Resource scenarios

\begin{tabular}{|l|l|l|l|l|}
\hline Scenario & Nr. Server type1 & Nr. Server type2 & Nr. Server type3 & Nr. Server type4 \\
\hline Modern -1 & 50 & 100 & 150 & 200 \\
\hline Normal -2 & 100 & 100 & 100 & 100 \\
\hline Old -3 & 200 & 150 & 100 & 50 \\
\hline
\end{tabular}

With each resource configuration, we generated a raw set of jobs. The jobs come randomly to the system within the period of 1000 time slots. The parameters for each job are determined by random selection. As the jobs in the traditional computing style scenario may have a long runtime period, we selected the runtime for each job spanning from 1 to 100 time slots. It should be noted that 1 time slot lasts 5 minutes.

We generated 3 federated data centers configurations: federated data centers with many old data centers, federated data centers with balanced types of data center, federated data centers with many modern data centers. The detail of each federated data centers configuration is presented in Table 6. 
Table 6. Configuration of three federated data centers

\begin{tabular}{|l|l|l|l|l|}
\hline Federated ID & Configuration & Nr. Old centers & Nr. Normal centers & Nr. Modern centers \\
\hline 1 & Many Old & 6 & 3 & 1 \\
\hline 2 & Balanced & 3 & 4 & 3 \\
\hline 3 & Many Modern & 1 & 3 & 6 \\
\hline
\end{tabular}

We use 3 PUE/CUE configurations as presented in Table 7.

Table 7. PUE/CUE configurations

\begin{tabular}{|l|l|l|l|l|}
\hline Type & Energy source & PUE & ESC & CUE \\
\hline Low & $\begin{array}{l}\text { Oil 20\%, Hydro 40\%, Nuclear } \\
40 \%\end{array}$ & 1.3 & 0.12844 & 0.166792 \\
\hline Medium & $\begin{array}{l}\text { Coal 50\%, Nuclear 30\%, Hydro } \\
20 \%\end{array}$ & 1.5 & 0.45983 & 0.689745 \\
\hline High & Coal 80\%, Oil 20\% & 1.8 & 0.85 & 1.53 \\
\hline
\end{tabular}

To assign PUE/CUE to data center, we use 3 assigning configurations as presented in Table 8.

Table 8. Assigning configurations

\begin{tabular}{|l|l|}
\hline Assign ID & Energy source \\
\hline 1 & $\begin{array}{l}\text { Old data center high PUE/CUE, normal data center normal PUE/CUE, modern data } \\
\text { center low PUE/CUE }\end{array}$ \\
\hline 2 & $\begin{array}{l}\text { Old data center normal PUE/CUE, normal data center normal PUE/CUE, modern data } \\
\text { center normal PUE/CUE }\end{array}$ \\
\hline 3 & $\begin{array}{l}\text { Old data center low PUE/CUE, normal data center normal PUE/CUE, modern data center } \\
\text { high PUE/CUE }\end{array}$ \\
\hline
\end{tabular}

With each federated data centers configuration, with each PUE/CUE configuration, we run 3 simulation scenarios.

- Scenario 1: Each new VM will be allocated with the single allocation algorithm developed in phase 1 (see D4.1), in its own data center

- Scenario 2: Each new VM will be allocated with single federated allocation algorithm (see Chapter III.1.1.a.).

- Scenario 3: Each new VM will be allocated with single federated allocation algorithm (see Section 3). Every $k$ timeslots, call global federated allocation algorithm.

For each scenario, calculate the Energy/CO $\mathrm{CO}_{2}$ in 1000 timeslots where 1 timeslot is an arbitrary unit, which simulates 5 minutes of real time. We compare the result of the three aforementioned scenarios.

\subsection{Results}

The simulation results in terms of energy consumption (in $\mathrm{MW} *$ timeslot) are presented in Table 9. 
Table 9. Simulation results for energy consumption

\begin{tabular}{|l|l|l|l|l|l|l|l|}
\hline \multirow{2}{*}{$\begin{array}{l}\text { Feder } \\
\text { ated }\end{array}$} & \multirow{2}{*}{$\begin{array}{l}\text { Assign } \\
\text { ID }\end{array}$} & $\begin{array}{l}\text { Single } \\
\text { allocation } \\
\text { Energy }\end{array}$ & - & \multicolumn{2}{l}{$\begin{array}{l}\text { Single federated } \\
\text { allocation }\end{array}$} & \multicolumn{2}{|l|}{ Single + global federated allocation } \\
\cline { 4 - 8 } & & Energy & Saving & Energy & Transfer Energy & Saving \\
\hline 1 & 1 & 385.79 & 277.049 & $28 \%$ & 265.6591 & 0.003442 & $31 \%$ \\
\hline 1 & 2 & 354.12 & 329.6796 & $7 \%$ & 313.7542 & 0.003736 & $11 \%$ \\
\hline 1 & 3 & 338.59 & 289.3345 & $15 \%$ & 275.3579 & 0.003646 & $19 \%$ \\
\hline 2 & 1 & 395.18 & 314.6082 & $20 \%$ & 301.7559 & 0.049073 & $24 \%$ \\
\hline 2 & 2 & 396.13 & 369.2494 & $7 \%$ & 354.1649 & 0.033214 & $11 \%$ \\
\hline 2 & 3 & 413.55 & 326.031 & $21 \%$ & 312.712 & 0.004585 & $24 \%$ \\
\hline 3 & 1 & 412.09 & 357.4885 & $13 \%$ & 343.6139 & 0.068402 & $17 \%$ \\
\hline 3 & 2 & 445.99 & 418.3728 & $6 \%$ & 402.1352 & 0.003558 & $10 \%$ \\
\hline 3 & 3 & 502.17 & 367.9638 & $27 \%$ & 353.6827 & 0.004252 & $30 \%$ \\
\hline
\end{tabular}

The energy saving rate achieved applying federated optimization compared to not applying it is also presented in Fig. 3 .

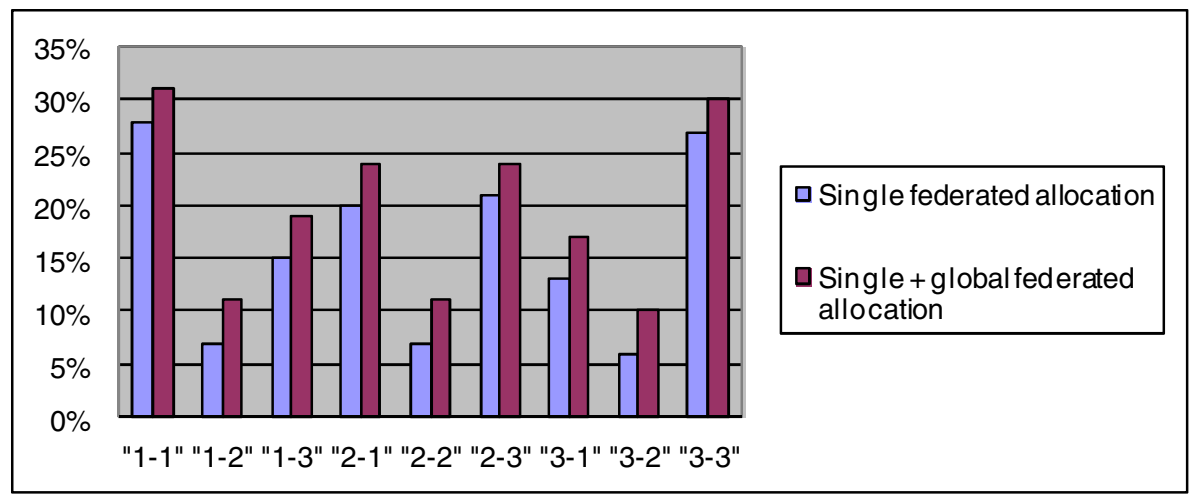

Fig. 3. Energy saving rate of applying federated optimization for traditional data centers

The simulation result in terms of $\mathrm{CO}_{2}$ emission (in Ton*timeslot/h) is presented in Table 10.

Table 10. Simulation results for $\mathrm{CO}_{2}$ emission.

\begin{tabular}{|l|l|l|l|l|l|l|l|}
\hline $\begin{array}{l}\text { Feder } \\
\text { ated }\end{array}$ & \multirow{2}{*}{$\begin{array}{l}\text { Assig } \\
\mathrm{n} \text { ID }\end{array}$} & & $\begin{array}{l}\text { Single } \\
\text { allocation } \\
\mathrm{CO}_{2}\end{array}$ & & $\begin{array}{l}\text { Single } \\
\text { allocation }\end{array}$ & & \multicolumn{2}{l|}{ federated } & \multicolumn{2}{l|}{ Single + global federated allocation } \\
\cline { 3 - 8 } & & $\mathrm{CO}_{2}$ & Saving & $\mathrm{CO}_{2}$ & \multicolumn{2}{l}{$\begin{array}{l}\text { Transfer } \\
\mathrm{CO}_{2}\end{array}$} & Saving \\
\hline 1 & 1 & 252.81 & 34.36192 & $86 \%$ & 32.94925 & 0.00279 & $87 \%$ \\
\hline 1 & 2 & 162.83 & 151.5919 & $7 \%$ & 144.2691 & 0.005537 & $11 \%$ \\
\hline 1 & 3 & 124.12 & 40.41604 & $67 \%$ & 38.46371 & 0.008822 & $69 \%$ \\
\hline 2 & 1 & 183.24 & 37.32418 & $80 \%$ & 35.79941 & 0.005822 & $80 \%$ \\
\hline 2 & 2 & 182.15 & 169.7897 & $7 \%$ & 162.8534 & 0.003441 & $11 \%$ \\
\hline 2 & 3 & 233.33 & 45.11798 & $81 \%$ & 43.27482 & 0.00734 & $81 \%$ \\
\hline 3 & 1 & 117.63 & 41.04069 & $65 \%$ & 39.44785 & 0.007853 & $66 \%$ \\
\hline 3 & 2 & 205.08 & 192.3807 & $6 \%$ & 184.9142 & 0.00496 & $10 \%$ \\
\hline 3 & 3 & 363.22 & 49.277 & $86 \%$ & 47.3645 & 0.006843 & $87 \%$ \\
\hline
\end{tabular}


The $\mathrm{CO}_{2}$ emission saving rate achieved applying federated optimization compared to not applying it is also presented in Fig.4.

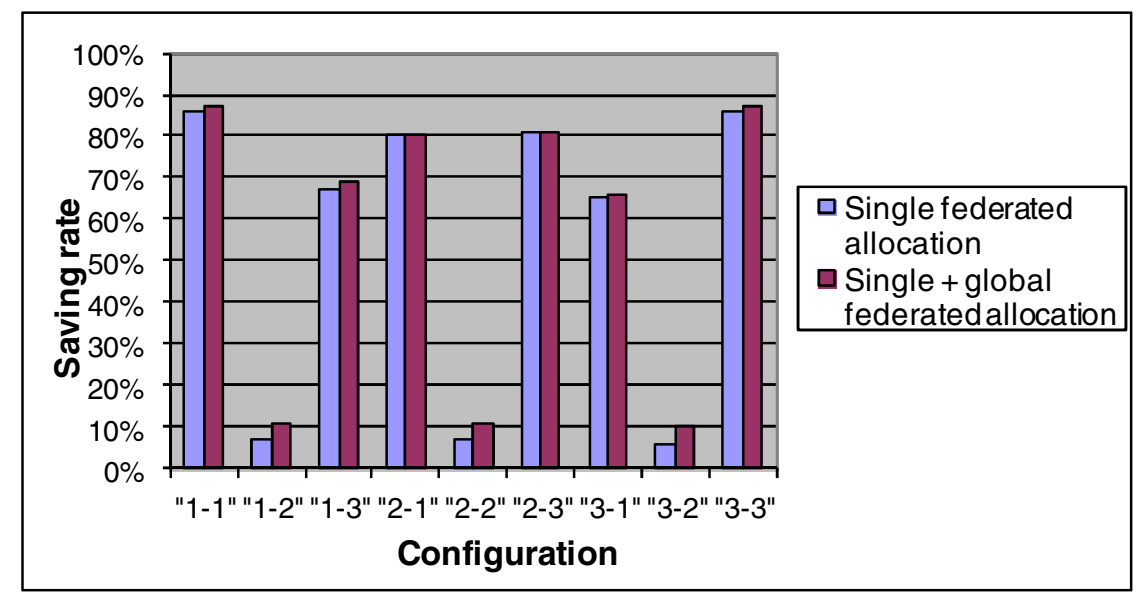

Fig. 4. $\mathrm{CO}_{2}$ emission saving of applying federated optimization for traditional data centers

The simulation result shows the efficiency of the energy aware algorithms for the federated traditional data centers. Depending on the configuration, the saving rate spans from $10 \%$ to $31 \%$ in energy consumption and from $10 \%$ to $87 \%$ in $\mathrm{CO}_{2}$ emission.

From the distribution of saving rate for both energy and $\mathrm{CO}_{2}$ emission according to configurations, we can see that the federated optimization algorithm is very effective when the values of PUE/CUE of each data center in the federation is greatly differ from each other. Indeed, with the simulation configurations 1-2, 2-2 and 3-2, where the PUE/CUE values are the same for each data center, the saving rate is much smaller than in other configurations where the PUE/CUE values have a large variance.

\section{Conclusions and Future Work}

In this work we have proposed the optimization algorithms for resources management in a federated data center. To decrease the power consumption and carbon emission, we needed to find the servers with the lowest power consumption and shift the workload to these facilities. Simulations made with different types and configurations of data centers have shown that the power consumption saving can be up to $31 \%$ and carbon emission reduction can be up to $87 \%$.

Our future work aims at applying the devised optimization algorithms in cloud data centers and evaluate the energy and carbon emission saving rates. 
Acknowledgements. The authors would like to acknowledge the FIT4Green project (grant agreement no. 249020) sponsored by the European Commission. The authors also thank Mikko Majanen (VTT, Finland) for his valuable comments.

\section{References}

1. Dang, M.-Q., Basmadjian, R., De Meer, H., Lent, R., Mahmoodi, T., Sannelli, D., Mezza, F., Dupont, C.: Energy efficient resource allocation strategy for cloud data centres. In: 26th Int. Symposium on Computer and Information Sciences, pp. 133-141. Springer Press (2011)

2. Berl, A., Gelenbe, E., Di Girolamo, M., Giuliani, G., De Meer, H., Dang, M.-Q., Pentikousis, K.: Energy-Efficient Cloud Computing. J. Computer 53(7), 1045-1051 (2010)

3. Bradley, D.J., Harper, R.E., Hunter, S.W.: Workload-based power management for parallel computer systems. IBM J. of Research and Development 47(5-6), 703-718 (2003)

4. Meisner, D., Gold, B.T., Wenisch, T.F.: PowerNap: Eliminating server idle power. In: 14th International Conference on Architectural Support for Programming Languages and Operating Systems, pp. 205-216. ACM Press (2009)

5. Carrol, R., Balasubramaniam, S., Donnelly, W.D.: Dynamic optimization solution for green service migration in data centres. In: IEEE International Conference on Communications, pp. 1-6. IEEE Press (2011)

6. Barbagallo, D., Di Nitto, E., Dubois, D.J., Mirandola, R.: A Bio-inspired Algorithm for Energy Optimization in a Self-organizing Data Center. In: Weyns, D., Malek, S., de Lemos, R., Andersson, J. (eds.) SOAR 2009. LNCS, vol. 6090, pp. 127-151. Springer, Heidelberg (2010)

7. Berral, J.L., Goiri, I., Nou, R., Julia, F., Guitart, J., Gavalda, R., Torres, J.: Towards energyaware scheduling in data centers using machine learning. In: 1st International Conference on Energy-Efficient Computing and Networking, pp. 215-224. ACM Press (2010)

8. Tang, Q., Gupta, S.K.S., Varsamopoulos, G.: Energy-efficient thermal-aware task scheduling for homogemeous high-performance computing data centers: a cyber-physical approach. IEEE Transactions on Parallel and Distributed Systems 19(11), 1458-1472 (2008) 